\title{
Aide humanitaire et production de services publics en Afrique de l'Ouest : le cas de la gestion des populations mauritaniennes refugiées au Sénégal
}

\section{Marion Fresia}

\section{(2) OpenEdition}

\section{Journals}

Édition électronique

URL : http://journals.openedition.org/apad/146

DOI : 10.4000/apad.146

ISSN : 1950-6929

Éditeur

LIT Verlag

\section{Édition imprimée}

Date de publication : 1 décembre 2002

\section{Référence électronique}

Marion Fresia, «Aide humanitaire et production de services publics en Afrique de l'Ouest : le cas de la gestion des populations mauritaniennes refugiées au Sénégal », Bulletin de l'APAD [En ligne], 23-24 | 2002, mis en ligne le 15 décembre 2006, consulté le 08 septembre 2020. URL : http:// journals.openedition.org/apad/146 ; DOI : https://doi.org/10.4000/apad.146

Ce document a été généré automatiquement le 8 septembre 2020

Bulletin de I'APAD 


\title{
Aide humanitaire et production de services publics en Afrique de l'Ouest : le cas de la gestion des populations mauritaniennes refugiées au Sénégal
}

\author{
Marion Fresia
}

1 Instable politiquement, et soumise aux caprices d'un climat difficile, l'Afrique sub-saharienne a connu au cours des années 90 de nouveaux mouvements forcés de populations. Elle a ainsi atteint un triste record: accueillir le tiers de la population réfugiée mondiale sur son territoire (HCR 2000) ${ }^{1}$. Les déplacements forcés ne sont pas nouveaux en Afrique, mais la lourdeur des interventions humanitaires qu'ils occasionnent aujourd'hui présente, par contre, un caractère inédit. Depuis la fin de la guerre froide en particulier, ce type d'intervention s'est multiplié et a impliqué un nombre croissant d'acteurs institutionnels et de moyens engagés. Cette évolution -liée à la fin de la paralysie du système des Nations-Unies et à la volonté des bailleurs de diversifier leurs canaux de distribution (Forster 1994) projettent les populations déplacées au cœur de dispositifs institutionnels complexes ayant pour mandat de les gérer, c'est à dire d'assurer leur installation, leur survie et leur devenir.

2 Cette situation n'a pas été sans susciter l'intérêt des milieux intellectuels et universitaires qui, dès les années 80 , se sont interrogés sur la légitimité de l'aide humanitaire et l'idéologie qui la sous-tend ${ }^{2}$. Il s'agissait, dans cette perspective, de souligner l'ambivalence du discours "humanitaire" qui, sous couvert d'humanisme, a pu servir à justifier le glissement de l'assistance vers l' "ingérence" et ainsi de poursuivre la coopération au développement par d'autres moyens (Perrot 1994 ; Rufin, 1994). Le débat s'est donc dès le départ posé dans des termes normatifs et a par la suite évolué vers des positionnements idéologiques décrivant l'humanitaire tour à tour comme espace d'oppression et de domination - un dispositif "déshumanisant" et 
"dépolitisant" - ou comme lieu d'expression de l'héroïsme du peuple, de ses capacités d'adaptation et de survie (Harell Bond \& Voutira 1992 ; Cernea \& Mc Dowell 2000 ; Zetter 1993 ; Allen 1996 ; Kibreab 1993) ${ }^{3}$.

3 Peu d'études ont finalement rendu compte du sens concret et empirique que revêt, au niveau local, une intervention humanitaire ${ }^{4}$. Or la simple observation montre que l'aide humanitaire constitue, avant tout, une offre de services "sociaux" 5 . dans des domaines aussi divers que l'eau, la santé, l'alimentation, l'éducation ou encore la sécurité. Les sites de réfugiés sont, de fait, toujours munis de leurs propres infrastructures (centre de santé, école, points d'eau, magasins de vivres) et dispositifs sécuritaires (Agier 2000). Les objectifs officiels de l'aide humanitaire sont d'ailleurs bien d'apporter protection juridique et assistance matérielle aux populations déplacées en attendant qu'une solution "durable" leur soit trouvée.

De ce constat émerge un ensemble de questions relatives à la dimension empirique d'une intervention humanitaire : comment les services proposés par l'aide humanitaire sont-ils mis en place dans le pays hôte? Quels acteurs institutionnels et non institutionnels participent à leur définition et leur réglementation? Comment ces services s'insèrent-ils dans les espaces socio-politiques et publiques locaux et que deviennent-ils après le désengagement des organisations humanitaires?

5 Seules des questions de ce type nous intéressent ici : elles nous permettent de donner un contenu tangible et non normatif à la notion d'humanitaire, et donc de comprendre comment, concrètement, des populations déplacées sont accueillies dans un pays hôte, et comment se gère au quotidien la pression qu'elles exercent sur ses infrastructures et ses ressources.

6 Cette perspective peut aussi mettre en évidence certains processus sociaux en cours dans les sociétés africaines, et ainsi constituer une porte d'entrée vers l'exploration d'autres objets sociologiques. En donnant quelques éléments de réponses aux interrogations soulevées ici, nous voudrions ainsi montrer en quoi l'aide humanitaire peut nous apporter un éclairage sur la notion de "gouvernance" au quotidien en Afrique, c'est à dire sur la manière dont les acteurs sociaux s'organisent pour assurer l'offre de services publics (Blundo 2002). L'aide humanitaire offrant des services sociaux apparemment semblables à des "services publics" ${ }^{6}$, il s'agira de se demander si elle se constitue ou non en espace de gouvernance.

7 Pour développer mon propos, je m'appuierai sur le cas de l'intervention humanitaire qui a suivi l'arrivée, en 1989, d'environ 70000 mauritaniens dans le Nord du Sénégal. Après avoir brièvement rappelé la spécificité de ces populations "réfugiées de longue date", j'analyserai à partir de deux études de cas la dynamique de production puis de transformation des services sociaux crées dans le cadre de cette intervention. Je tenterai de montrer comment ces services ont entraîné une reconfiguration de l'espace public local pour enfin m'interroger sur les rapports entre aide humanitaire et gouvernance locale.

Les réfugiés mauritaniens au Sénégal : "des réfugiés de longue date"

8 En 1989, des milliers de Mauritaniens ont été expulsés de force de leur pays suite à un différend frontalier qui opposa le Sénégal et la Mauritanie ${ }^{7}$.

9 A cette date, le gouvernement mauritanien procéda non seulement au rapatriement de 50000 sénégalais résidant sur son territoire mais aussi à la déportation par milliers de citoyens mauritaniens "noirs". 
10 le long de la vallée du fleuve Sénégal ${ }^{8}$. Contraints de traverser la frontière, ils ont trouvé refuge sur la rive gauche du fleuve, peuplée par leurs parents proches, des Halpulaar de nationalité sénégalaise. Dans un premier temps, leur prise en charge a été assurée par la Croix Rouge sénégalaise, une diversité d'ONG et d'associations locales, ainsi que par les populations autochtones elles-mêmes. Cependant, lorsque le nombre des mauritaniens affluant au Sénégal a dépassé le seuil des 10 000, le gouvernement sénégalais a fait appel au système des Nations-Unies. Les représentants des différentes branches onusiennes (HCR, PNUD, OMS, PAM, UNICEF) ont alors organisé une mission conjointe d'évaluation des besoins et sont tombés d'accord sur la mise en place d'un "programme d'urgence". Ce programme devait être coordonné par le HCR et mis en œuvre par des ONG sélectionnées par celui-ci comme "partenaires opérationnels". Pour que le mandat du HCR puisse s'exercer, l'Etat sénégalais devait préalablement reconnaitre officiellement l'existence de réfugiés mauritaniens sur son territoire. Ainsi, par décret ministériel, a-t-il reconnu de prima facie (prime abord) le statut de réfugié à l'ensemble des populations mauritaniennes ayant franchi la frontière pour venir au Sénégal ${ }^{9}$.

11 Les réfugiés ont alors été installés dans plus de 280 sites dispersés le long de la vallée du fleuve Sénégal près de la frontière mauritanienne. Certains réfugiés, originaires d'un même village, ont pu se regrouper à nouveau et négocier le lieu de leur installation, généralement proche de leurs parents sénégalais. D'autres ont été contraints ou ont choisis de s'installer dans des sites sélectionnés préalablement par le HCR et les autorités locales, selon des critères de proximité aux voies de communication, aux infrastructures collectives et/ou aux services publics sénégalais.

12 Après la période d'urgence, qui a duré environ une année, le HCR a continué à encadrer et porter une assistance matérielle aux réfugiés mauritaniens jusqu'en juillet 1998. Plusieurs volets d'intervention étaient prévus: distribution de vivres, installations hydrauliques, santé et hygiène, enseignement et activités génératrices de revenus. Concrètement, cette aide a débouché sur la création, dans la vallée, de 18 nouvelles cases de santé et d'une quarantaine de salles de classe de niveau primaire réparties entre différents sites; des bornes fontaine et des latrines ont par ailleurs été aménagées systématiquement.

13 Pour gérer ces nouvelles infrastructures et répartir les vivres, les réfugiés se sont aussi organisés au sein des sites. Ils ont mis en place leur propre administration interne avec des Présidents ou chefs de "sites", des "délégués" de quartiers, et en formant des commissions ou caisses pour la santé, l'eau, les vivres etc. Les projets de soutien économique ont par ailleurs favorisé l'émergence de nombreux groupements féminins et des groupements d'intérêts économiques mixtes (GIE) ${ }^{10}$ dans les domaines de l'agriculture et du maraîchage.

14 Depuis 1998, le HCR n'intervient plus que dans deux domaines: la "protection juridique" des réfugiés, et la recherche de "solutions durables" ${ }^{11}$. Ainsi, treize ans après leur expulsion, les sites de réfugiés mauritaniens sont toujours en place, ils forment des villages que l'on distingue difficilement du reste du paysage sénégalais. Si beaucoup sont rentrés en Mauritanie, ou d'autres ont immigré vers les villes, ils reviennent en réalité régulièrement dans les sites où leurs femmes et leurs enfants sont restés et ils continuent à y investir leurs ressources. 
15 Les populations mauritaniennes sont donc des "réfugiés de longue date". Ils se trouvent paradoxalement installés sur leur propre territoire historique ${ }^{12}$, une situation loin d'être exceptionnelle en Afrique. Leur cas apparaît cependant assez singulier, si l'on s'attache aux modalités d'intervention du HCR. Comparativement à d'autres opérations menées sur le continent (Soudan, Somalie, région des grands lacs), celle-ci est en effet de faible ampleur, et relativement souple. L'assistance délivrée n'a pas occasionné la formation de camps comptant des milliers de réfugiés - les sites sont petits, entre 50 et 2000 personnes et dispersés sur une distance de plus de $500 \mathrm{~km}$ - ni la construction d'infrastructures "lourdes", tels des hôpitaux ou des routes. De plus, elle a laissé une marge de manœuvre importante aux réfugiés quant aux choix de leur mode d'installation et d'administration interne.

Cependant, même lorsque l'aide humanitaire n'engage pas de gros moyens, elle contribue à modifier l'espace public local. Elle favorise l'émergence de nouveaux acteurs sociaux qui se positionnent autour de l'opposition identitaire et juridique "réfugié"/"non-réfugié", et ceci alors même que les populations en question peuvent être de proches parents.

17 Comme ailleurs, mais à une autre échelle, l'aide humanitaire s'est constituée en enjeu important au Sénégal, et a fait l'objet de diverses formes de récupérations politiques et économiques. C'est ce que je voudrais souligner à travers une analyse concrète de la mise en œuvre de deux des volets de l'intervention humanitaire au Sénégal : la santé et l'enseignement. Le fait qu'il s'agisse de "réfugiés de longue date" permet d'adopter une perspective diachronique et d'observer comment ces services ont évolué, en particulier après le désengagement du HCR en matière d' "assistance matérielle".

Production et transformation de deux services sociaux : la santé et

l'enseignementNaissance de cases de santé et de salles de classes dans les sites de réfugiés

La santé et l'éducation étaient des volets prévus par le programme d'assistance du HCR. Cependant, leur mise en place ne relève pas de la seule décision du HCR. Il existe en pratique des processus de négociations complexes entre divers acteurs sociaux que nous voulons ici mettre en évidence ${ }^{13}$.

Prenons le cas du site de regroupement de Ndioum, dans le département de Podor : il comptait en 1989 environ 2000 personnes. Pendant la phase d'urgence, un "embryon" de service sanitaire avait été mis en place par la Croix Rouge Sénégalaise avec le soutien de Médecins du Monde. Il s'agissait de soins primaires dispensés gratuitement sur le site, mais aussi de dons réguliers en médicaments dont seuls les réfugiés pouvaient bénéficier sur présentation de leur carte de réfugié ${ }^{14}$. Après plus d'une année de gratuité totale des soins, Médecin du Monde, devenu partenaire opérationnel du HCR pour la santé, accepte, à la demande des représentants du site, de financer la construction d'une case de santé et de la doter de matériel et de médicaments. Les représentants des réfugiés, comptant parmi eux des infirmiers diplômés d'Etat et nombre de fonctionnaires, allaient ainsi pouvoir prendre en charge eux-mêmes la gestion de la case et exercer une activité professionnelle.

L'ONG pose cependant plusieurs conditions aux réfugiés. Elle exige, d'une part, leur participation au financement et à la réalisation matérielle du projet, et d'autre part un système de recouvrement des coûts à partir d'une dotation initiale en médicaments. Les représentants du site acceptent mais négocient en retour une rémunération du personnel réfugié médical et des membres du futur comité de santé. Ils obtiennent ainsi 
des indemnités salariales allant de 250000 à 500000 F CFA : elles leur seront versées par le HCR jusqu'en 98. Une fois le système en place, Médecins du Monde se désengage, et le HCR confie le "suivi" du volet santé à l'OFADEC, une ONG sénégalaise. Celle-ci se propose dès lors de mieux "structurer" le système mis en place par le partenaire précédent : elle emploie un médecin consultant chargé d'aider le comité de santé à s'approvisionner, et donc l'encadrer dans sa gestion. Elle cherche également de nouvelles dotations gratuites en médicaments. Concernant les règles d'accessibilité aux soins, elles sont fixées par le comité de santé : la case est ouverte aux autochtones comme aux réfugiés, et les médicaments sont vendus à prix égal pour tous à des tarifs nettement inférieurs que ceux pratiqués par les structures sanitaires sénégalaises. Cependant seuls les autochtones paient la consultation mais à un prix fixé très bas. C'est selon ce même processus que les 17 autres cases de santé ont été mises en place et structurées de façon assez similaire dans d'autres sites de la vallée du fleuve.

21 Le cas de l'enseignement est assez semblable, si ce n'est la part d'initiative prise par les réfugiés qui est plus importante. En effet, les représentants des réfugiés, qui comptaient parmi eux des enseignants, ont mobilisé les populations pour construire des écoles où serait enseigné le programme mauritanien (comprenant une composante arabe), ceci afin de ne pas perturber la scolarité de leurs enfants. Ils ont réussi à réunir des cotisations et à trouver un financement près d'ONG locales pour construire d'éphémères salles de classe, où ils exerçaient au départ leur profession volontairement. Ils avaient également mis en place par le biais de l'association des réfugiés mauritaniens sénégalais (ARMS) un système interne de coordination des programmes entre écoles de réfugiés et de formation de réfugiés souhaitant enseigner bénévolement. Ces efforts ont alors servi de vitrine pour négocier auprès de la délégation du HCR à Dakar un financement couvrant la livraison de matériel scolaire, la construction de nouvelles classes et le paiement d'indemnités salariales aux enseignants. La gestion de ce fond a, par la suite, été confiée à l'OFADEC, qui, comme pour la santé, s'est chargé de mieux structurer et de maintenir le système mis en place par les représentants des réfugiés en employant un "coordinateur" pour l'enseignement et en faisant la "promotion" des réfugiés mauritaniens pour obtenir d'autres fonds à distribuer. Ainsi une quarantaine de classes primaires sont nées dans la vallée. Conçues dans une optique du retour, elles se sont paradoxalement inscrites dans la durée.

Remarquons qu'elles n'accueillaient que des enfants réfugiés étant donné les programmes enseignés.

Ces infrastructures scolaires et sanitaires présentent donc comme caractéristiques communes d'avoir été mises en place et financées selon un processus hybride, à coup de négociations entre ONG partenaires du HCR (OFADEC, Médecins du Monde), représentants des réfugiés (chefs de sites, comités pour la santé, ou pour l'éducation), et populations réfugiées elles-mêmes (participation de type "communautaire"). Leurs règles de fonctionnement et d'accessibilité ont été largement fixées par les représentants de réfugiés eux-mêmes (ARMS, comités de gestion) mais sous les conseils et avec l'encadrement de consultants engagés par les ONG partenaires. Elles étaient en effet directement supervisées par lesdits consultants, le personnel enseignant et sanitaire devant leur remettre régulièrement des rapports d'activité (financier et épidémiologique pour les cases de santé, de suivi scolaire pour les écoles). Les autorités administratives sénégalaises locales (médicales et de l'enseignement), quant à elles, 
n'assuraient qu'une supervision "indirecte". Selon leurs propres termes, les infrastructures des sites de réfugiés avaient "un statut à part", elles étaient à "80\% indépendante" et relevaient de "l'autorité du HCR" ; certains représentants parlent même d'écoles ou de cases de santé d'un "troisième type" pour en souligner la spécificité par rapport au système sénégalais. Il semble d'ailleurs qu'aucun document officiel n'existe pour préciser de manière claire le statut et les règles de fonctionnement de ces infrastructures, que ce soit au niveau de l'administration sénégalaise ou du projet HCR-OFADEC.

Ces constats nous amènent dès lors à formuler quelques remarques importantes quant aux logiques qui ont présidé la mise en place de ces services sociaux :

Intermédiation et courtage

La construction de cases de santé et de salles de classes a été principalement négociée par des acteurs sociaux qui font office d'intermédiaires entre les populations réfugiées d'une part, et les bailleurs de fonds de l'autre (le HCR) :

- Il y a d'une part les représentants des réfugiés, des "intellectuels" issus de l'administration publique mauritanienne. Ils se sont organisés et regroupés au sein de "bureaux de sites", et $\mathrm{d}$ "'associations de réfugiés", officiellement à caractère social, à l'abri desquelles ils mènent aussi des activités politiques. Leur statut de "porte-parole" des réfugiés et leur position d'intermédiaire leur offrent la possibilité et la légitimité de mettre en œuvre des logiques de courtage auprès des organisations humanitaires pour obtenir une assistance maximale. Cette logique se traduit aussi par des stratégies de captage de ressources (petits détournements) ou de redistribution ciblée de ces mêmes ressources afin de former des réseaux de clientèle politique.

- Il y a d'autre part les ONG intermédiaires, partenaires ou agences d'exécution du HCR. L'OFADEC apparaît ici jouer un rôle important, non pas tellement dans la production mais dans le maintien et la structuration des services sociaux nés pendant la phase d'urgence. A partir de 1992, elle est devenue de fait le seul partenaire du HCR chargé de suivre et assurer l'exécution de tous les volets de l'assistance matérielle. Le partenariat se fonde sur des accords annuels qui confèrent à l'OFADEC la tâche de gérer les fonds du HCR selon un programme fixé normalement par celui-ci. En pratique cependant, l'ONG dispose d'une marge de manœuvre significative qui va bien au-delà de son rôle d'exécutant. Occupant le terrain en permanence, elle fait des propositions et recommandations au HCR quant à la définition de ses programmes et peut ainsi suggérer des réorientations budgétaires.

L'OFADEC et les représentants des réfugiés ont finalement des pratiques et des intérêts convergents qui produisent des services sociaux et les inscrivent dans la durée. Ces acteurs vivent tous deux de l'existence même d'une situation humanitaire et donc de la présence de "réfugiés"; ils en tirent des ressources économiques mais aussi une légitimité sociale. Pour les fonctionnaires, s'ajoute une dimension politique puisque l'existence de sites de réfugiés alimente aussi leur discours de dénonciation du régime mauritanien, renforce leur combat politique clandestin et leur sert de base pour leurs réseaux de clientèle.

Cette convergence d'intérêts se manifeste aussi par l'utilisation, de la part de ces intermédiaires, d'un même registre discursif de type "humanitariste" qui vise à "mettre en scène" la situation extrêmement difficile endurée par les réfugiés ${ }^{15}$. Leurs discours contribuent ainsi à légitimer et concrétiser la catégorie juridique du "réfugié", et leurs actions de courtage permettent de drainer des investissements importants vers les 
sites, en matière de santé et d'enseignement, et ainsi de les constituer en espaces sociaux durables.

Attentisme de l'Etat

Dans cette dynamique est remarquable la quasi-absence de l'Etat. Si les autorités administratives locales étaient présentes pendant la phase d'installation des réfugiés, il semble qu'entre 1990 et 1996 elles ne jouent qu'un rôle indirect, du moins dans la mise en place des infrastructures nées de l'intervention humanitaire. Sans moyens pour intervenir en matière d'assistance aux réfugiés, l'Etat s'est en réalité retiré volontairement.

L'Etat profite ainsi de la situation : ne pouvant faire face à la pression exercée par les populations arrivantes sur ses infrastructures et services publics, il laisse le HCR financer et agir à sa place, ce qui lui permet de faire d'énormes économies ${ }^{16}$. C'est bien ce que l'Etat souhaitait en 1989 lorsqu'il a fait appel aux systèmes des Nations-Unies et a reconnu officiellement l'existence de réfugiés sur son territoire. Par cet acte, il délègue, de fait, ses compétences en matière d'offre de services publics, ce qui explique qu'il considère dès lors les réfugiés comme une population relevant de la seule autorité du HCR dans ce domaine. Cette position contribue à renforcer l'opposition "réfugié/ non réfugié", les uns et les autres n'étant pas, semble-t-il, placés sous la même autorité, alors même qu'ils sont sur le même territoire. Il faut ici préciser que cette position varie bien sûr en fonction des enjeux. A d'autres moments et pour d'autres raisons, les autorités revendiqueront clairement l'exercice de leur mandat sur les populations réfugiées. Dans le domaine de la protection juridique par exemple, après le rétablissement de ses relations diplomatiques avec la Mauritanie en 1992, l'Etat sénégalais fera manifestement blocage au HCR pour délivrer des cartes de résidence de 10 ans aux réfugiés - ceci pour des raisons essentiellement politiques. Nous verrons aussi comment l'Etat tentera aussi de reprendre progressivement contrôle sur la gestion des infrastructures construites en milieu réfugié, lorsque celles-ci commenceront à concurrencer les services publics sénégalais.

Les services sociaux mis en place sous l'impulsion d'une dynamique de courtage des intermédiaires semblent finalement servir l'intérêt d'un grand nombre d'acteurs. En effet, les populations réfugiées bénéficient d'accès aux soins et à l'enseignement à moindre coût, les autochtones profitent de leur côté d'un accès aux soins à des tarifs avantageux (mais aussi à d'autres infrastructures des sites tels les robinets d'eau), enfin l'Etat lui-même tire avantage de la situation en laissant son territoire s'enrichir de nouvelles infrastructures sans engagement financier de sa part. Ainsi, est-il possible d'affirmer que ces services sont d' "intérêt général" mais sans pour autant relever du secteur public, c'est-à-dire sans que l'Etat n'ait participé à la définition de leur statut, de leur implantation ni de leurs règles de fonctionnement. Nous sommes donc dans une configuration particulière où des intérêts privés collectifs (ceux des intermédiaires et des réfugiés), d'ordre économique et politique, débouchent sur la création de services profitables à tous, et où l'intérêt général se définit en dehors des autorités étatiques.

31 Il s'agit dès lors de se demander comment ces services évoluent sur le long terme, en particulier lorsque leurs bailleurs (le HCR) - qui font aussi vivre les intermédiaires décident de se désengager.

Le désengagement du HCR et le devenir des services de santé et d'enseignement Considérant les réfugiés comme "auto-suffisants", d'un niveau de vie "équivalent" à celui des autochtones, et subissant par ailleurs certaines pressions politiques, le HCR 
décide de se désengager progressivement à partir de 1995 du volet "assistance matérielle" de son programme ${ }^{17}$.

Cette politique commence par l'arrêt définitif de la distribution de vivres en 1995, et se poursuit par la volonté de mettre progressivement fin à l'assistance portée aux réfugiés dans les domaines de l'enseignement et de la santé. Dès 1996, pour préparer cette éventualité, le HCR pose aux autorités administratives sénégalaises la question de 1"'intégration" des écoles de réfugiés et des cases de santé dans le système administratif sénégalais. Par intégration, il est question de savoir si l'Etat sénégalais est prêt à rémunérer le personnel réfugié selon des modalités à définir, et à assurer la supervision directe des cases de santé et des écoles en adaptant leurs normes de fonctionnement à celles du système sénégalais.

Pour répondre à cette question, les administrations locales négocient dans un premier temps la possibilité de faire "une tournée" des cases et des écoles - tournée financée par le HCR - afin d'évaluer la compétence du personnel réfugié, le matériel manquant et l'effectif des populations fréquentant ces infrastructures. La mission aboutit à un ensemble de constats critiques de la part de l'inspection départementale de l'enseignement, et de la supervision du district sanitaire: le personnel réfugié est accusé de mauvaise gestion ou d'incompétence, et le matériel est déclaré incomplet ou obsolète.

Finalement, en juillet 98, lorsque le HCR décide l'arrêt du versement des indemnités salariales aux réfugiés, et se désengage officiellement de toute supervision de ces infrastructures, l'Etat accepte le principe de l'intégration mais l'applique de manière sélective.

Concernant les écoles tout d'abord, si les enfants sont "intégrés" au système scolaire sénégalais, les professeurs réfugiés eux ne le sont pas, et se retrouvent donc au chômage. Les salles de classes sont soit fermées et le matériel transféré vers une école sénégalaise, ou bien, elles restent ouvertes mais des professeurs sénégalais y sont affectés. Si l'intégration des élèves réfugiés représente de nouvelles charges pour l'Etat, celles-ci sont compensées par les résultats scolaires plutôt faibles des enfants réfugiés, qui vont rarement au delà de la 6eme, et par le fait que les directeurs d'école obtiennent des dons extérieurs facilement en mettant en avant que leurs écoles accueillent de nombreux enfants réfugiés.

Quant aux cases de santé, elles sont intégrées au système sanitaire sénégalais dans le sens où elles se trouvent désormais directement supervisées par les districts sanitaires départementaux. Elles doivent cependant fonctionner selon un mode "communautaire", l'Etat refusant de prendre en charge le personnel réfugié. Les infirmiers réfugiés sont ainsi maintenus mais à condition que les populations puissent les rémunérer elles-mêmes à partir des recettes de la vente des médicaments et des tickets de consultation (de même pour la matrone et les membres du comité de gestion). Ce nouveau mode de gestion est ainsi venu rétrograder le statut des infirmiers réfugiés qui, bien que pour la plupart diplômés d'Etat, se retrouvent être rémunérés tels des agents communautaires de santé.

En instaurant ce système, l'Etat laisse en réalité jouer les lois de la concurrence : là où l'affluence est importante, le personnel réfugié arrive à obtenir une rémunération suffisante pour accepter de rester. C'est le cas des cases de santé situées dans des sites de réfugiés éloignés de structures sanitaires sénégalaises: la hausse des prix des médicaments et des consultations entraînées par la fin du système de subvention n'a 
pas impliqué une baisse de la fréquentation ; n'ayant pas d'autre choix, réfugiés comme autochtones continuent à venir s'y soigner. Par contre, les cases situées à proximité de dispensaires autochtones se voient mises à mal.

Alors qu'auparavant, elles concurrençaient ceux-ci du fait de leurs tarifs avantageux, la situation se trouve inversée: ce sont désormais les dispensaires autochtones qui récupèrent la clientèle réfugiée mais aussi sénégalaise. Aussi, ces cases soit fonctionnent au ralenti sur financement communautaire, soit sont fermées du fait du départ de l'infirmier réfugié non remplacé. Dans ce dernier cas - deux cases ont fermé à ce jour - le matériel a été récupéré par l'Etat et transféré vers la structure sénégalaise la plus proche.

Reclassement des services produits vers le secteur public

Ainsi avec le désengagement du HCR, assiste-t-on à une logique de reclassement des services sociaux nés de l'intervention humanitaire vers le secteur public; c'est en effet désormais à l'Etat qu'il appartient de fixer les règles de fonctionnement des cases de santé et de superviser et encadrer leurs comités de gestion, c'est également lui qui prend en charge la scolarité des enfants réfugiés. Dans la mesure où l'Etat n'a pas participé financièrement à la mise en place de ces services, il est donc possible de parler d'une stratégie de récupération d'infrastructures sociales produites dans une configuration dont il était absent. Cette récupération se fait, de plus, à moindre coût, puisque l'Etat privilégie un mode de gestion communautaire pour les cases de santé d'une part, et refuse l'intégration des enseignants réfugiés dans le système éducatif sénégalais d'autre part.

41 Les populations réfugiées ont très mal réagi à cette situation qui implique une hausse des coûts des soins et de scolarité. Surtout, elles considèrent que les infrastructures construites étaient avant tout pour "eux", et à "eux". Ils avaient cotisé et participé à leur financement, et à leur fonctionnement ; le personnel était des leurs, il était "très compétent et attirait tous les villages environnants par sa compétence et sa disponibilité", comme le répètent les habitants de Ndioum. Selon eux, l'Etat sénégalais leur a ainsi "volé leurs biens" et ceci avec la complicité du HCR et du gouvernement mauritanien.

Quant aux représentants des réfugiés, mis au chômage ou démotivés par leurs faibles rémunérations, ils décident de réorienter leur logique de courtage vers le volet "protection juridique" qu'assure toujours le HCR. Ainsi, mettant en avant leur absence de perspective professionnelle mais aussi leur insécurité liée à leurs positions politiques "gênantes" pour le gouvernement sénégalais, ils mènent des démarches pour se faire "réinstaller" ou obtenir l'asile politique dans les pays occidentaux. Plus de 200 d'entre eux réussissent ainsi, en novembre 2000, à s'envoler vers les Etats Unis où ils bénéficient de formations linguistiques et professionnelles pour s'y installer durablement. Beaucoup étaient des infirmiers et des enseignants qui travaillaient dans les structures des sites de réfugiés.

43 Ainsi, sur le long terme, les services sociaux nés de l'intervention humanitaire sont-ils intégrés dans le secteur public sénégalais alors même que, de manière contradictoire, ils semblent ne plus servir l'intérêt général.

44 En effet, les populations, réfugiées comme autochtones, ne bénéficient plus de soins à moindre coût. La gestion désormais purement "communautaire" des cases de santé semble ne pas fonctionner et suscite absentéisme et détournements de médicaments ou de fonds de la part des infirmiers démotivés ${ }^{18}$. Les écoles voient de leur côté leurs 
effectifs gonflés par l'arrivée des enfants réfugiés, alors qu'elles étaient déjà pléthoriques.

Enfin, la nécessité de payer les fournitures scolaires oblige nombre de familles réfugiées à renoncer à scolariser leurs enfants. Le départ des fonctionnaires pour les Etats-Unis a par ailleurs suscité un certain "désarroi" dans les sites, les réfugiés ayant l'impression d'être "abandonnés et oubliés de tous". Elle a également entraîné des ressentiments parmi les autochtones qui veulent aussi partir aux Etats-Unis. Ceux-ci tentent pour cela d'acheter aux réfugiés leurs cartes ou de se faire délivrer de fausses cartes. L'enjeu de la réinstallation a ainsi encouragé le développement d'un trafic de cartes dans la vallée qui avait déjà commencé au moment de la distribution des vivres - et alimenté les pratiques corruptives de l'administration en matière de délivrance de papiers d'identité.

Aide humanitaire et reconfiguration de l'espace public local

En faisant appel à une assistance extérieure pour faire face à un afflux de populations sur son territoire, nous avons vu comment l'Etat sénégalais s'est vu obligé de reconnaître collectivement le statut de réfugiés aux mauritaniens. Cette reconnaissance a dès lors placé légalement ces populations sous mandat du HCR, mandat qui s'exerce automatiquement sur toute personne dite réfugiée au sens des Conventions de Genève de 1951 et des Protocoles d'accord de 1967. Elle a entraîné, suivant des logiques d' intermédiation et de courtage, la production et surtout le maintien dans la durée d'un ensemble de services sociaux d'intérêt général situés en dehors du secteur public. L'Etat en effet ne décide ni ne supervise la mise en place et le fonctionnement de ces services; il ne participe pas non plus à leur financement ou à leur gestion ${ }^{19}$. Dans un premier temps, cette situation semble favorable à beaucoup d'acteurs sociaux: elle permet aux administrations publiques de faire d'énormes économies, et offre aux réfugiés, mais aussi aux autochtones, des services sociaux gratuits ou à moindre coûts. Cependant, son maintien au delà de la période d'urgence semble, par la suite, devenir problématique.

Tout d'abord, les services sociaux mis en place par l'aide humanitaire concurrencent progressivement l'espace public local. De manière inattendue, ils ont été structurés tels de véritables services publics satisfaisant les intérêts du plus grand nombre. Subventionnés par des organismes "plus riches" que l'Etat (le HCR et autres bailleurs), les comités de gestion de ces services peuvent pratiquer des tarifs plus bas que ceux des structures publiques sénégalaises. Comme expliqué précédemment, les cases de santé ont ainsi pu attirer la clientèle autochtone en la détournant des dispensaires sénégalais.

Ensuite, l'émergence d'une nouvelle forme de citoyenneté liée au statut de réfugié va également venir concurrencer l'espace public qui ne peut la contrôler qu'indirectement. En effet, si les autorités publiques sont les seules à pouvoir, en principe, reconnaître le statut de réfugié aux concernés, les droits et devoirs liés à ce statut sont négociés ailleurs, au niveau intergouvernemental. Ce statut, parce qu'il facilite l'accès aux services proposés par l'humanitaire, tels la santé mais aussi l'eau, la distribution gratuite de vivres, les activités génératrices de revenus, et la réinstallation dans les pays occidentaux, constitue un enjeu non seulement pour les réfugiés qui veulent le maintenir, mais aussi pour les autochtones qui tentent de l'acquérir par des moyens informels et illégaux. Cette situation "gêne" aussi l'Etat mais à un niveau plus macro-politique, car depuis le rétablissement de ses relations diplomatiques avec la 
Mauritanie, en 1992, il subit des pressions de la part de son homologue mauritanien pour étouffer l'existence de réfugiés mauritaniens sur son sol.

En autorisant le HCR à exercer ses fonctions, l'Etat a donc lui-même contribué à l'émergence sur son territoire d'une sorte d'espace "extra territorial" : celui des sites de réfugiés et de leurs habitants. A l'image des missions diplomatiques, l'Etat n'a qu'un contrôle très indirect sur ce qui se passe à l'intérieur de cet espace et sur les droits de ses "ressortissants". Cependant, il ne reste pas sans réagir à cette concurrence et cet embarras que lui cause le maintien - sans doute inattendu - sur le long terme de cet espace "humanitaire". Aussi tente-t-il de le replacer progressivement sous son autorité. D'une part, cela s'exprime au niveau central par un manque de volonté politique manifeste de reconnaître aux réfugiés mauritaniens un statut individuel durable, mais aussi par un contrôle plus strict du renouvellement de ces cartes pour éviter que des "autochtones" s'y infiltrent. D'autre part, avec le désengagement du HCR, que l'Etat a pu en partie influencer, nous avons vu comment cette logique de "reconquête" se traduit au niveau local par la récupération au sein du secteur public, et à moindre coûts, des services mis en place par les représentants des réfugiés et les organismes partenaires du HCR.

Ce repositionnement de l'Etat et du HCR a pour conséquence de remodeler à nouveau l'espace public local en y intégrant de nouvelles populations que les administrations de la santé et de l'éducation doivent désormais gérer. La mise au chômage du personnel réfugié, par un Etat qui a privilégié ses intérêts, a par ailleurs entraîné une nouvelle dynamique de renforcement du statut de réfugié. Aujourd'hui, les Mauritaniens du Sénégal sont plus que jamais résolus à négocier le maintien de leur statut de réfugié du fait des enjeux liés aux perspectives d'émigration, ce qui ne les empêche pas, par ailleurs, d'obtenir de manière frauduleuse des papiers sénégalais afin par exemple d'exercer librement certaines activités professionnelles (commerce) et de circuler plus facilement.

51 Le gouvernement sénégalais, et sans doute le HCR, n'avaient pas prévu cette situation. Tous deux espéraient que l'arrêt de l'assistance matérielle allait pousser les populations réfugiées soit à rentrer en Mauritanie, soit à prendre officiellement la nationalité sénégalaise. Ainsi, contrairement à ce qui était souhaité, le "dossier" des réfugiés mauritaniens n'est toujours pas clos et se complexifie même. En effet, en pratique, les réfugiés cumulent à la fois, au niveau familial ou individuel, leur carte provisoire de réfugié, leurs papiers mauritaniens récupérés grâce à des parents bien placés en Mauritanie, et des papiers sénégalais, obtenus de la même manière, grâce à des parents sénégalais. Leur terroir s'inscrivant de part et d'autre de la frontière, ils continuent ainsi, comme ils l'ont toujours fait, d'aller et venir de part et d'autres de la frontière en fonction des opportunités et ressources disponibles en jouant sur un cumul des identités citoyennes ${ }^{20}$. Ces pratiques viennent donc "brouiller" les cartes, et rendent plus difficile l'action des autorités gouvernementales ou intergouvernementales.

52 Finalement, l'intervention humanitaire a eu de multiples "effets inattendus" : bien que construite sur des principes de l'apolitisme et du transitoire ${ }^{21}$, elle s'inscrit en pratique dans la durée et a des conséquences politiques réelles dans le champ public local. L'assistance matérielle et juridique du HCR proposée a, en effet, empiété de fait sur les domaines de compétence de l'Etat en suscitant l'émergence d'une nouvelle offre de services publics et d'une nouvelle citoyenneté concurrençant ou chevauchant celles de l'Etat. Dans un dernier point, je montrerai en quoi ces effets "inattendus" sont de 
l'ordre de la "gouvernance" et peuvent donc apporter un certain éclairage sur cette notion.

L'humanitaire, espace de gouvernance?

53 La "gouvernance" est un concept né aux Etats-Unis dans un contexte où les autorités locales semblaient avoir de plus en plus du mal à "gouverner" à elles seules une société toujours plus atomisée et complexifiée en sous-systèmes et réseaux divers capables de résister à leurs injections. Face à ce problème de gouvernementalité", des mécanismes alternatifs de coordination se seraient mis en place pour rendre possible l'action publique, la suppléer ou du moins la rendre plus efficace; ce sont ces modes "nouveaux" de régulation que la notion de gouvernance se propose de décrire et de recouvrir; elle suppose de sortir d'une vision institutionnelle, rationnelle et standardisée de l'action du gouvernement pour en observer ses manifestations informelles, et éclatées. Elle s'intéresse ainsi aux formes opportunistes et flexibles de l'action publique; et aux dynamiques de négociation entre divers acteurs sociaux gouvernementaux et non gouvernementaux qui tentent, sur le mode du bricolage, de la rendre possible et efficace ${ }^{22}$.

54 Si l'on considère l'action publique sous l'angle de l'offre de services publics, il nous semble dès lors qu'une intervention humanitaire constitue un lieu privilégié d'expression et d'observation de la notion de gouvernance. Elle constitue en effet l'un de ces modes alternatifs de coordination qui produisent de manière assez inattendue de "l'action publique", en dehors de l'autorité étatique ou du moins en ne l'impliquant qu'indirectement.

ete à partir d'une étude de cas, nous avons observé comment au sein de l'espace humanitaire sont nés des services d'intérêt général, situés hors du secteur public, à partir de logiques d'intérêts privés, d'intermédiation et de courtage, ayant des objectifs à la fois politiques et économiques. Dans ce processus, l'Etat a certes joué un rôle en "autorisant" le HCR à intervenir. Cependant il n'était qu'un acteur parmi tant d'autres à participer à la mise en place d'une action "publique" qui visait à répondre à l'afflux de populations déplacées. Inversement, nous avons par ailleurs constaté qu'il peut y avoir des formes d'action publique produites par l'autorité étatique mais qui ne servent pas l'intérêt général. Ainsi la logique de récupération par l'Etat des services produits par l'intervention humanitaire répondait-elle avant tout à la satisfaction des intérêts des fonctionnaires de l'Etat qui voyaient leurs structures se faire concurrencer.

Cette logique de récupération a par ailleurs permis de mettre en valeur le caractère mouvant et non figé de l'offre de services d'intérêt général, qui peut, en fonction du moment, des enjeux et du positionnement des différents acteurs, relever du secteur public ou non.

57 Des acteurs privés peuvent donc se rallier à des actions d'intérêt général alors que des acteurs publics peuvent utiliser des méthodes de gestion privée ou se comporter tels des entrepreneurs. La "gouvernance" implique donc bien un déplacement ou un questionnement des barrières entre privé et public.

Si cette notion décrit des modes alternatifs, nouveaux ou hybrides qui visent à assurer l'action publique, il faut ajouter qu'ils ne la rendent pas pour autant plus efficace en matière de "gouvernementalité", c'est a dire en matière de gestion rationnelle d'une population donnée obéissant aux principes de l'économie politique et usant des techniques d'un appareil sécuritaire (Foucault 1991). Les populations que visent ces modes de gouvernement ne sont, en effet, pas pour autant mieux contrôlées ou dirigées 
d'un point de vue politique et économique. Pour en revenir à notre cas, les négociations entre le HCR et l'Etat sénégalais, visant progressivement a faire disparaitre l'existence officielle de refugiés dans la vallée du fleuve, n'ont par exemple pas eu les effets escomptés. La légitimité du statut de réfugié s'est au contraire renforcée et a d'autant plus complexifié des dynamiques citoyennes locales déjà éclatées entre la Mauritanie et le Sénégal.

Finalement, au delà de la rhétorique officielle du provisoire et de la neutralité, l'espace humanitaire apparaît bien comme un espace de gouvernance. Les enjeux liés a l'assistance matérielle et la protection juridique des refugiés font naître des formes d'action publique aux contours flous et jamais définitifs. Mises en œuvre par une diversité d'acteurs, privés comme publics, ces actions semblent relever bien plus du bricolage et de réajustements permanents que d'une logique rationnelle et calculée visant à un contrôle efficace des populations réfugiées. Gouvernance ne veut donc pas dire "gouvernementalité" au sens foucauldien du terme, parce que les logiques des acteurs s'enchâssent toujours dans des réalités sociales et historiques qui les préexistent et qui en modifient évitablement la portée et les objectifs.

\section{BIBLIOGRAPHIE}

Agier M., 2000, La guerre, le camp, la ville : enquête dans les camps de réfugiés de Dadaab au Kenya. Rapport de mission IRD/MSF-B, Centre d'études africaines, EHESS.

Allen T., (ed.), 1996, In search of cool grounds : war, flight and homecoming in northeast Africa. Geneva, New York, Africa World Press, United Nation research institute for social development.

Bergbeder Y., 1999, Haut Commissariat des Nations Unies pour les réfugiés, Coll. Que sais-je ?, Paris, PUF.

Blundo G., 2002, "La gouvernance au quotidien en Afrique, les relations entre services publics et collectifs et leurs usagers", introduction au colloque de l'APAD/ASC sur La gouvernance au quotidien en Afrique, Leyden, 22-25 mai.

Cernea M. et Mc. Dowell C. (eds.), 2000, Risks and reconstruction. Experiences of resettlers and refugees, Washington, DC, The World Bank.

Forster J., 1994, "Raisons d'Etat et raison humanitaire", in Perrot M.D, Dérives humanitaires, Etats d'urgence et droit d'ingérence, Coll. "Les nouveaux cahiers de l'IUED", Genève, Paris, IUED-PUF : 63-75.

Foucault M., 1991, "Governmentality" in Burchell G., Gordon C. \& Miller P., The Foucault Effect : Studies in Governmentality, Chicago, University of Chicago Press : 87-104.

Fresia M., 2001, L'expérience d'un déplacement forcé. Les modes de réappropriation de la contrainte dans le village de réfugiés mauritaniens de Ndioum au Sénégal, mémoire de DEA, EHESS-Marseille.

Goffman E., 1979, La mise en scène de la vie quotidienne. La présentation de soi. Paris, Ed. minuit. 
Grignon C. et Passeron J-C., 1989, Le savant et le populaire. Misérabilisme et populisme en sociologie et en littérature, Paris, Seuil.

Harrell-Bond B.E. et Voutira E., 1992, "Anthropology and the study of refugees", Anthropology today, vol. 8, nº $4: 6-10$.

HCR., 2000, Les réfugiés dans le monde. Cinquante ans d'action humanitaire, Paris, Ed. Autrement / HCR.

Kibreab G., 1993, "The myth of dependency among camp refugees in Somalia : 1979-1989", Journal of refugee studies, vol. $6, \mathrm{n}^{\circ} 3: 521-349$.

Lasailly-Jacob, Marchal J.Y., et Quesnel A. (dir.), 1999, Déplacés et réfugiés : la mobilité sous contrainte, Paris, IRD éditions.

Le Galès P., 1995, "Du gouvernement des villes à la gouvernance urbaine", Revue française de science politique, vol. 45, $\mathrm{n}^{\circ} 1: 57-96$.

Perrot M.D., 1994, Dérives humanitaires. Etats d'urgence et droit d'ingérence. Coll. "les nouveaux cahiers de l'IUED", Paris, Genève.

Rutin J.C., 1994, L'aventure humanitaire, Paris, Gallimard.

Sall M., 2000. Territorialisation, crises et réponses aux crises dans la moyenne vallée du fleuve Sénégal : une lecture à partir de la question des réfugiés Mauritaniens au Sénégal, Mémoire de DEA, Institut d'études du développement, Louvain.

Sall M., 2002, "Approche explicative de l'accès aux ressources publiques par les populations transfrontalières : une lecture à partir de la question des réfugiés mauritaniens dans la vallée du fleuve Sénégal", communication présentée au colloque de l'APAD/ ASC sur La gouvernance au quotidien en Afrique. Les relations entre services publics et leurs usagers, Leyden, 22-25.

Santoir C., 1990, "Le conflit mauritano-sénégalais : la genèse", Cahiers des sciences humaines, ORSTOM, vol. 26, $\mathrm{n}^{\circ} 4: 553-576$.

Schmitz J., 1986, "L'Etat géomètre : les leydi des Peul du Fuuta Toro (Sénégal) et du Massina (Mali)", Cahiers d'études africaines, vol 26, n $3: 349-394$.

Zetter R., 1991, "Labelling refugees : forming and transforming an identity", Journal of refugee studies, vol. $4, \mathrm{n}^{\circ} 1: 39-63$.

\section{NOTES}

1.Par mouvements de populations forcés, j'entends ici des mouvements massifs occasionnés par une crise politique et/ou une catastrophe naturelle ; selon le droit international, il est question de "réfugiés" seulement lorsqu'il y a franchissement d'une frontière internationale ; on parlera dans le cas contraire de "déplacés internes". Dans la présente contribution, je traiterai essentiellement des populations réfugiées.

2.Voir Perrot (1994). Cet ouvrage collectif et interdisciplinaire est un bon exemple des réflexions qui ont été menées jusqu'à présent sur la question de l'aide humanitaire et de sa légitimité.

3.J'ai montré dans le cadre d'un mémoire de DEA comment la littérature anthropologique anglo-saxonne relative au thème du déplacement forcé présente une certaine tendance à sombrer dans les pièges du misérabilisme ou du populisme idéologique tels que Grignon et Passeron les ont définis (1989). 
4.Il faut noter ici l'ouvrage de Lasailly-Jacob, Marchal, et Quesnel (dir.) (1999), qui se situe dans une telle perspective empirique.

5.J'emploie volontairement le terme de "services sociaux" à ce stade de la présentation, car l'une des réflexions centrales de cette contribution est bien de comprendre si ces services doivent être considérés comme publics (étatiques), ou collectifs (privés). 6.J'entends ici par services publics, des services destinés à servir l'intérêt général, celui-ci étant en principe défini par des autorités étatiques.

7.En avril 1989, dans la moyenne vallée du fleuve Sénégal, près de Diawara, un incident frontalier entre cultivateurs soninke sénégalais et éleveurs peuls mauritanien cause la mort de deux personnes. L'incident sert de prétexte au déclenchement d'une spirale de violence à Nouakchott puis à Dakar entre Sénégalais et Mauritaniens et à la rupture des relations diplomatiques entre les deux pays. Pour une analyse des causes du conflit, voir Santoir (1990).

8.Il y avait parmi les expulsés des wolof et des soninke également, mais la majorité était formée par des Halpulaar peuplant la moyenne et la haute vallée du fleuve Sénégal. Certains des réfugiés ont aussi été expulsés vers le Mali mais n'ont pas fait l'objet d'assistance humanitaire significative. Enfin d'autres ont été expulsés par voie aérienne et sont arrivés à Dakar avant d'être transférés dans les sites de réfugiés de la vallée du fleuve.

9.Cette procédure est utilisée en cas de déplacements massifs de population, qui nécessite une intervention rapide et ne laisse pas le temps d'octroyer le statut de réfugié au cas par cas, de manière individuelle. Elle est à distinguer de l'obtention d'une reconnaissance individuelle du statut de réfugié qui donne plus de droits en matière de libre circulation et qui seule permet l'obtention du droit d'asile ou du droit à la réinstallation dans un pays tierce (autre que le premier pays d'accueil).

10.Par GIE mixte, il est fait référence à des groupements composés d'un tiers d'autochtones et de deux tiers de réfugiés. Ce principe de mixité avait été proposé par l'ONG partenaire du HCR, OFADEC, pour éviter d'éventuels conflits fonciers avec les autochtones.

11.Ces domaines sont en réalité la raison d'être du HCR. Selon ses statuts, sa mission principale est $d$ "'assumer des fonctions de protection internationale (...) et de recherche de solutions permanentes au problème des réfugiés" (art 1). Cependant, pour mener à bien cette mission, le HCR a de fait développé une seconde fonction d'assistance opérationnelle et matérielle aux réfugiés qui est la plus visible. Le HCR distingue trois types de solutions durables : le rapatriement volontaire dans le pays d'origine, l'intégration dans le pays hôte, la réinstallation dans un pays tiers. Pour plus de détails à ce sujet, voir Bergbeder (1999).

12.Les populations Halpulaar ont toujours habité de part et d'autre du fleuve Sénégal, leurs territoires politiques (de la dynastie peu le des Deniankoobe au $16^{\mathrm{e}}$ siècle au régime théocratique des Almaami au $19^{\mathrm{e}}$ siècle) mais aussi économiques (zones agro-pastorales) se situaient avant la colonisation à cheval entre les deux rives. C'est avec les indépendances seulement que certains sont devenus "Mauritaniens" et d'autres "Sénégalais". Sur la formation de ces territoires (leydi) transversaux au fleuve, voir Schmitz (1986).

13.Les études des cas présentées ici sont tirées de recherches en cours que je mène dans divers sites de réfugiés mauritaniens dans le département de Podor ; elles se basent sur des entretiens menés avec les populations réfugiées, leurs représentants, les populations autochtones. les autorités administratives locales (sous-préfet. inspecteur 
départemental de l'enseignement. médecin chef de district sanitaire), les représentants du HCR et de l'OFADEC.

14.Bien qu'ayant fait l'objet d'une reconnaissance de prima facie, les réfugiés mauritaniens ne disposent pour la plupart que d'un "récépissé de demande" d'obtention du statut de réfugié, valable trois mois (renouvelable) ; cette carte fait office de "pièce d'identité" provisoire et permet d'obtenir l'accès aux services sociaux proposés par le HCR.

15.Par l'expression de "mise en scène", je fais surtout référence au travail de $\mathrm{E}$, Goffman (1979). Il ne s'agit pas ici de minimiser la douleur endurée par les populations réfugiées qui est, au contraire, un sentiment qui participe pleinement de certaines logiques d'action mises en œuvre par celles-ci.

16.Précisons ici que selon le droit international, c'est normalement aux Etats qu'il incombe d'assister matériellement les réfugiés, le HCR n'ayant pour mission essentielle que de leur assurer leur protection juridique. Cependant, les Etats des pays du "Sud" n'ayant pas les moyens suffisants pour assurer cette tâche, le HCR a vu sa mission s'étendre à ce domaine là aussi (Bergbeder 1999).

17.Les raisons officielles de ce désengagement sont liées à l'autosuffisance constatée des réfugiés. Cependant, le HCR a sans doute subi certaines pressions de la part des gouvernements mauritanien et sénégalais qui depuis le rétablissement de leurs relations diplomatiques préfèrent occulter l'"affaire" des réfugiés mauritaniens. 18.Remarquons que les détournements étaient aussi pratiqués pendant la période de l'engagement du HCR, cependant ils étaient moins visibles du fait de l'activité des cases de santé qui fonctionnaient bien et permettaient à tous de se soigner.

19.Que l'Etat délègue le financement et la gestion de services publics à des opérateurs non étatiques ne doit pas étonner, c'est une pratique courante qui n'enlève rien au caractère "public" du service, c'est à dire au fait que le service soit défini par l'Etat comme devant servir l'intérêt général.

20.Au sujet des stratégies transfrontalières mises en œuvre par les réfugiés pour assurer leur accès aux services publics, voir le mémoire de Sali (2000) ainsi que sa contribution au colloque de l'Apad (2002).

21.Selon le statut du HCR, "L'activité du HCR ne comporte aucun caractère politique : elle est humanitaire et sociale" (art 2) ; le caractère temporaire de son activité est par ailleurs inscrit dans son mandat qui est de 5 ans, renouvelable par l'Assemblée générale des Nations Unies ; ainsi depuis 1951, le HCR voit-il son mandat renouveler tous les cinq ans (Bergbeder 1999).

22.Ce paragraphe s'inspire largement de l'article de Le Galès (1995).

\section{AUTEUR}

\section{MARION FRESIA}

Allocataire de recherche de l'Ecole des hautes études en sciences sociales, marfresia@yahoo.fr 